\title{
Gout in the thoracic spine causing acute paraplegia: illustrative case
}

\author{
Lacin Koro, BA, ${ }^{1}$ Ryan Khanna, MD, MBA, MSCl, ${ }^{1}$ Dominick Richards, BA, ${ }^{1}$ and Dean G. Karahalios, $\mathrm{MD}^{2}$ \\ ${ }^{1}$ Department of Neurological Surgery, Rush University Medical Center, Chicago, Illinois; and ${ }^{2}$ Brain \& Spine Institute, Advocate Aurora Health, Downers Grove, Illinois
}

BACKGROUND Although spinal involvement by gout is not uncommon, spinal gout leading to symptomatic spinal cord compression in the thoracic spine is very rare and poses a diagnostic challenge by mimicking symptoms of more common diagnoses such as epidural abscess and malignancy. An even more unique presentation is spinal gout causing thoracic cord compression leading to acute paraplegia.

OBSERVATIONS The authors present an illustrative case of a 35-year-old man with thoracic spinal compression by tophaceous gout who developed rapid progression to complete paraplegia over a 5-day period. Magnetic resonance imaging of the thoracic spine revealed a cystic-appearing lesion within the dorsal extradural space of the lower thoracic spine extending from T8 to T10 accompanied by compression of the spinal cord. An emergent T9-10 laminectomy was performed, and the occupying lesion in the thoracic spine was resected. The diagnosis of spinal tophaceous gout was made by pathological examination.

LESSONS Although varying clinical manifestations of spinal gout have been reported in the literature, the patient's age and the rapid progression to complete paraplegia over a 5-day period reveals a unique presentation that broadens understanding of the manner in which this condition can present and allow more rapid diagnosis and treatment.

https://thejns.org/doi/abs/10.3171/CASE21308

KEYWORDS spinal gout; tophus; thoracic spinal cord compression; acute paraplegia

Gout is a metabolic disease characterized biochemically by the deposition of monosodium urate crystals. When the extracellular concentration of uric acid exceeds the human physiological urate solubility threshold, urate crystals develop and commonly form deposits in various joints, bursa synovialis, subcutaneous tissue, and the urinary tract. The usual manifestations are crystal arthropathy and tophi deposition in the soft tissues. Although gout is prevalent worldwide, reported cases of spinal gout are less frequently reported and present variably with acute, subacute, or chronic symptoms. Patients commonly present with back pain and neurological impairments often mimicking the symptoms of more common diagnoses such as epidural abscess, spondylodiscitis, and malignancy. ${ }^{1}$ Although various clinical manifestations of spinal gout have been reported in the literature, this condition places a disproportionately greater burden on older adults with an extensive history of gout and rarely manifests with rapidly progressing paraplegia on initial presentation. ${ }^{1}$ We report a case of a 35 -year-old male with thoracic spinal cord compression by tophaceous gout who developed progressive spastic paraplegia and lower extremity numbness acutely over a 5-day period.

\section{Illustrative Case \\ History and Examination}

A 35-year-old male with a past medical history of gout and hypertension presented with acute spastic paraplegia and bilateral lower extremity numbness. The patient had initially experienced focal lower back pain while he was lifting a heavy object 5 days before his arrival at the emergency department. Over the next several days, the patient experienced progressive paraparesis and gradual progression of bilateral lower extremity paresthesias below the umbilicus. He had no bowel dysfunction but was unable to void the evening before his arrival at the hospital, and he denied any previous history of surgery or trauma to the brain and spinal cord.

A neurological examination revealed normal strength, tendon reflexes, and sensation with no Hoffman sign or pronator drift of his bilateral upper 
extremities. His bilateral lower extremity strength was 0/5 except for spontaneous nonvoluntary dorsiflexion. Tenderness to palpation was found over the bilateral thoracic and lumbar paraspinal muscles starting at the thoracolumbar junction. Decreased sensation was observed starting at the level of the umbilicus and extending bilaterally to the lower extremities, and this was accompanied by perianal numbness and reduced rectal tone. The patient had bilateral hyperreflexia of his patellar tendon reflexes. He also had bilateral clonus of his Achilles tendons, and his bilateral Babinski's sign was positive. The initial laboratory test results were notable for an erythrocyte sedimentation rate (ESR) of $76 \mathrm{~mm} / \mathrm{hr}$ and a C-reactive protein (CRP) concentration of $11 \mathrm{mg} / \mathrm{L}$. A serum uric acid test was not ordered on admission. Other initial laboratory test results, which included a complete blood count and basic metabolic profile, were within the normal range.

Magnetic resonance imaging without contrast of the thoracic and lumbar spine revealed a complex, multilobulated, cystic-appearing $1.3-\mathrm{cm} \times 1.3-\mathrm{cm} \times 6.5-\mathrm{cm}$ lesion within the dorsal extradural space of the lower thoracic spine extending from the top of T8 to the bottom of the T10 level with associated compression of the thoracic spinal cord (Figs. 1 and 2).

\section{Operation, Pathological Findings, and Postoperative Course}

On the basis of his clinical symptoms and imaging, the patient underwent an emergent T9-10 laminectomy. Operative findings showed a dorsal epidural mass that grossly appeared to resemble a calcified chronic abscess and phlegmon that was densely adherent to the dura. The cystic mass was filled with milky fluid. The mass was dissected off the dura and sent for microbiological and histopathological examinations. After the surgery, the infectious disease service was consulted for concern of an epidural abscess, and the patient was started on empirical intravenous cefepime and vancomycin. The histopathological examination revealed red-pink bone with attached white chalky material resembling gouty tophi. Fragments of the mass stained with $100 \%$ alcohol were positive for urate crystals. A serum uric acid level was ordered and was elevated at 9.4 $\mathrm{mg} / \mathrm{dL}$. The empirical antibiotic treatment was discontinued in view of the consistent clinical, histopathological, and laboratory findings suggestive of spinal tophaceous gout, and the patient was started on colchicine and febuxostat. He was fitted for a custom orthosis and mobilized with
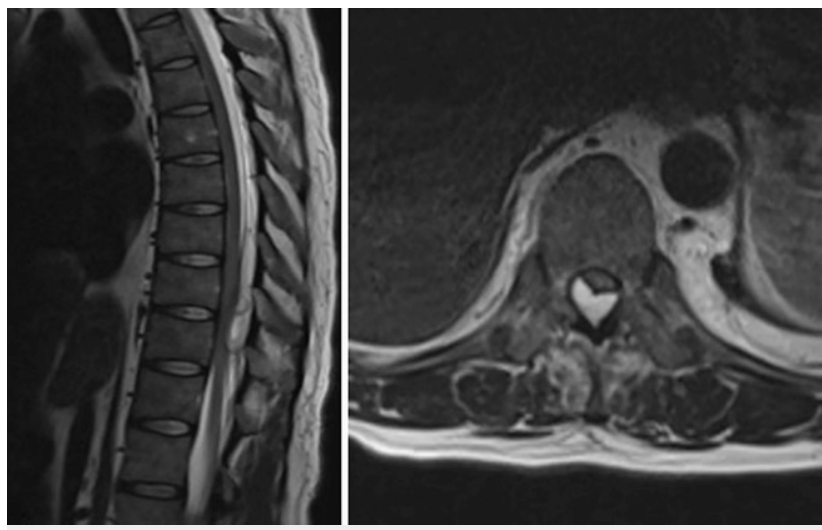

FIG. 1. Preoperative magnetic resonance imaging of the thoracic spine. Left: Sagittal plane T2-weighted section. Right: Axial plane T2weighted section. Images show a cystic-appearing lesion within the dorsal epidural thoracic spinal canal at T8-10 accompanied by extrinsic compression of the dorsal surface of the thoracic spinal cord that was most pronounced at $\mathrm{T} 10$.
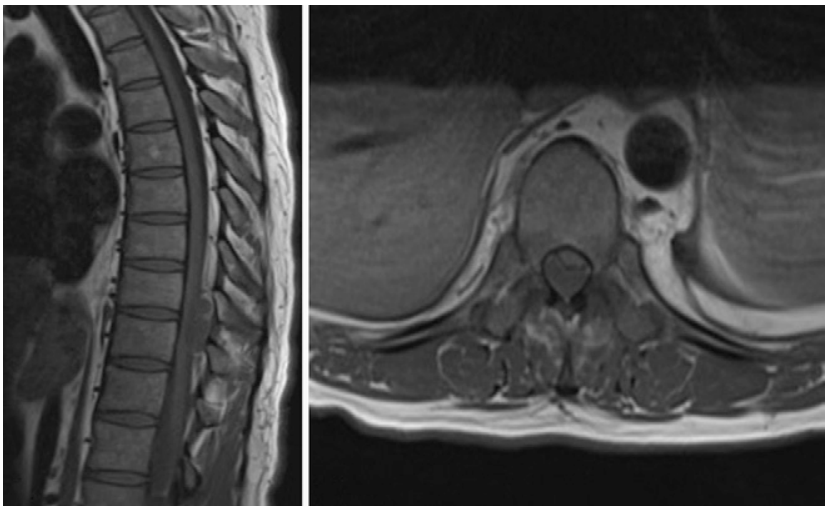

FIG. 2. Preoperative magnetic resonance imaging of the thoracic spine. Left: Sagittal plane T1-weighted section. Right: Axial plane T1weighted section. T1-weighted images without contrast show a cysticappearing lesion with intermediate signal intensity within the dorsal epidural thoracic spinal canal at T8-10 accompanied by extrinsic compression of the dorsal surface of the thoracic spinal cord that was most pronounced at T10.

physical therapy. By postoperative day 1 , he had already recovered significant motor function in his lower extremities. At the time of discharge, he had not developed any additional complications and was transferred for acute inpatient rehabilitation. Three months after admission, the patient had regained full strength in his lower extremities and reported only moderate pain with intermittent paresthesias, thus demonstrating significant neurological improvement as compared with his initial presentation.

\section{Discussion \\ Observations}

This case report describes a rare initial clinical manifestation of spinal tophaceous gout causing acute-onset and rapidly progressive spastic paraplegia in a 35-year-old male with a prior history of gout. Our case demonstrates the difficulty in accurately diagnosing spinal gout in a patient whose clinical presentation, demographics, and onset of symptoms mimic more common pathological entities such as epidural abscess and spondylodiscitis.

\section{Lessons}

Gout is a common metabolic disease that has been estimated to affect approximately $4 \%$ of all adults in the United States. ${ }^{2}$ However, clinically significant involvement of the spine is rare and poses a diagnostic challenge because the clinical manifestations of this condition have not been consistently characterized. Toprover et al. ${ }^{1}$ reviewed the characteristics of 131 previously reported cases of spinal involvement in gout and found that gout can involve the spine at any level. In their study, they found that axial gout involved the lumbar spine $(38 \%)$, cervical spine (24.8), and thoracic spine (17.8\%), respectively. ${ }^{1}$ In $19.4 \%$ of cases, more than one spinal region was involved. The duration of symptoms was not consistent and ranged from 1 day to 6 years, affecting a male-predominant $(76 \%)$ patient population with a median age of 58.9 years, with most cases occurring between ages 44 and 74 years. The symptoms with which patients presented were not consistent. The most common symptom reported was back pain, which was present in $68.5 \%$ of cases. The second most common complaint was 
neurological impairment, such as radiculopathy, loss of sensation, motor weakness, or paraparesis, affecting $65.4 \%$ of patients. In addition, Toprover et al. ${ }^{1}$ reported patients most commonly had an extensive history of gout or hyperuricemia as well as elevated uric acid levels and inflammatory markers such as ESR, CRP, and white blood cell count.

Although spinal involvement by gout is not uncommon, spinal gout causing thoracic spinal cord compression by tophus has been reported far less frequently. In 2018, Ding et al. ${ }^{3}$ reviewed the characteristics of 30 previously reported cases of thoracic spinal cord compression caused by tophaceous gout and found that at the onset of disease, $60 \%$ of patients presented with back pain and $43.3 \%$ had weakness and/or numbness in their lower limbs. Ding et al. concluded that clinical suspicion of spinal gout should be high if a patient with a medical history of prior gout or hyperuricemia presents with chronic and/or acute back pain and neurological symptoms. Despite this, the probability of spinal tophaceous gout leading to symptomatic spinal cord compression in the thoracic spine is very rare compared with other etiologies, such as neoplasm and infection. An even more unique presentation is tophaceous gout causing thoracic spinal cord compression leading to acute paraplegia, as seen in our case. To the best of our knowledge, there are only a handful of reported cases of acute paraplegia secondary to thoracic cord compression by spinal tophi. Koskoff et al. ${ }^{4}$ reported a case of a 44-year-old male with a previous history of alcoholism, tophaceous gout, and multiple episodes of gouty arthritis who presented with tophaceous gout of the spine causing spinal cord compression at the T9-11 levels. Their patient developed insidiously progressive weakness leading to the paralysis of his legs over a 4month period. Popovich et al. ${ }^{5}$ reported a case of a 36 -year-old woman with a previous history of uncontrolled tophaceous gout who had spinal tophaceous gout causing thoracic spinal cord compression. She presented with absence of sensory and motor function of both legs of 2 weeks' duration. Finally, Ibrahim et al. ${ }^{6}$ reported a case of thoracic spinal cord compression at the T1-2 levels caused by gouty arthropathy in a 70-year-old woman with a 1-year history of progressive weakness in her hands and lower extremities who presented with upper motor neuron paralysis of her legs.

Although varying clinical manifestations of spinal gout have been reported in the literature, our patient's age and his rapid progression to complete paraplegia over a 5-day period is a unique presentation that broadens our understanding of the manner in which this condition can present. Although spinal gout has been reported previously at ages near that of our patient, the symptoms in those patients were less severe and progressed less rapidly. ${ }^{7-13}$ Our case would suggest that an insidious progression of this clinical condition is not assured and that expeditious treatment may be most appropriate. The best management of cases in which spinal gout presents with neurological symptoms is rapid surgery for pathological diagnosis and decompression with subsequent pharmacological treatment to decrease uric acid levels. ${ }^{1}$

We hope that our case allows clinicians to better understand the variety of ways in which axial gout can present and leads to more rapid diagnosis and treatment to mitigate the morbidity associated with this condition.

\section{References}

1. Toprover M, Krasnokutsky S, Pillinger MH. Gout in the spine: imaging, diagnosis, and outcomes. Curr Rheumatol Rep. 2015;17(12): 70-77.

2. Zhu Y, Pandya BJ, Choi HK. Prevalence of gout and hyperuricemia in the US general population: the National Health and Nutrition Examination Survey 2007-2008. Arthritis Rheum. 2011;63(10): 3136-3141.

3. Ding Y, Wang W, Jiang W, Zhang L, Wang T, Li Z. Tophaceous gout causing thoracic spinal cord compression: case report and review of the literature. Neurochirurgie. 2018;64(3):171-176.

4. Koskoff YD, Morris LE, Lubic LG. Paraplegia as a complication of gout. J Am Med Assoc. 1953;152(1):37-38.

5. Popovich T, Carpenter JS, Rai AT, Carson LV, Williams HJ, Marano GD. Spinal cord compression by tophaceous gout with fluorodeoxyglucose-positron-emission tomographic/MR fusion imaging. AJNR Am J Neuroradiol. 2006;27(6):1201-1203.

6. Ibrahim GM, Ebinu JO, Rubin LA, de Tilly LN, Spears J. Gouty arthropathy of the axial skeleton causing cord compression and myelopathy. Can J Neurol Sci. 2011;38(6):918-920.

7. Kao Y, Wang Z, Leng J, et al. Thoracic gout tophus with abdominal wall protrusion: a case report. Medicine (Baltimore). 2020;99(10): e19348.

8. Leaney BJ, Calvert JM. Tophaceous gout producing spinal cord compression. Case report. J Neurosurg. 1983;58(4):580-582.

9. Wang Y, Zhu T, Wang D, et al. A case of thoracic cord compression due to tophi. Zhonghua Yi Xue Za Zhi. 2015;95:2880-2881.

10. Lu F, Jiang J, Zhang F, Xia X, Wang L, Ma X. Lumbar spinal stenosis induced by rare chronic tophaceous gout in a 29-year-old man. Orthopedics. 2012;35(10):e1571-e1575.

11. Jegapragasan M, Calniquer A, Hwang WD, Nguyen QT, Child Z. A case of tophaceous gout in the lumbar spine: a review of the literature and treatment recommendations. Evid Based Spine Care J. 2014;5(1):52-56.

12. Saripalli K, Baskar S. Tophaceous gouty arthropathy of the lumbar spine. Clin Med (Lond). 2014;14(6):683-684.

13. Mishra R, Panigrahi VP, Adsul N, et al. Tophaceous gout in thoracic spine mimicking meningioma: a case report and literature review. Surg Neurol Int. 2020;11:364.

\section{Disclosures}

The authors report no conflict of interest concerning the materials or methods used in this study or the findings specified in this paper.

\section{Author Contributions}

Conception and design: Karahalios, Koro, Khanna. Acquisition of data: all authors. Analysis and interpretation of data: Karahalios, Koro, Khanna. Drafting the article: Koro, Khanna. Critically revising the article: all authors. Reviewed submitted version of manuscript: all authors. Approved the final version of the manuscript on behalf of all authors: Karahalios. Study supervision: Karahalios.

\section{Correspondence}

Dean G. Karahalios: Brain \& Spine Institute, Advocate Aurora Health, Downers Grove, IL. dean.karahalios@aah.org. 